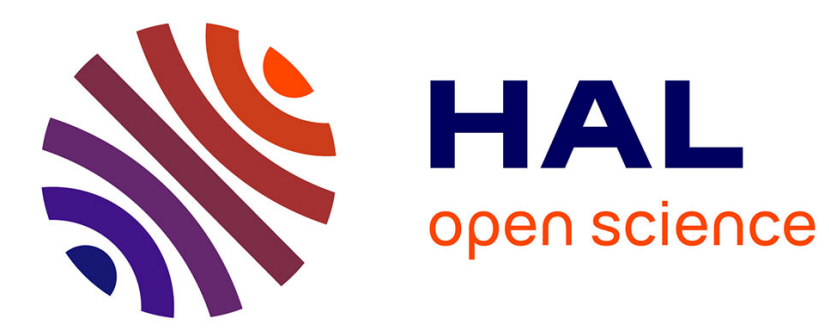

\title{
On the regularity of the inverse jacobian of parallel robots
}

\author{
Jean-Pierre Merlet, Peter Donelan
}

\section{To cite this version:}

Jean-Pierre Merlet, Peter Donelan. On the regularity of the inverse jacobian of parallel robots. ARK, Jun 2006, Ljubljana. inria-00001085

\section{HAL Id: inria-00001085 \\ https://hal.inria.fr/inria-00001085}

Submitted on 2 Feb 2006

HAL is a multi-disciplinary open access archive for the deposit and dissemination of scientific research documents, whether they are published or not. The documents may come from teaching and research institutions in France or abroad, or from public or private research centers.
L'archive ouverte pluridisciplinaire HAL, est destinée au dépôt et à la diffusion de documents scientifiques de niveau recherche, publiés ou non, émanant des établissements d'enseignement et de recherche français ou étrangers, des laboratoires publics ou privés. 


\title{
ON THE REGULARITY OF THE INVERSE JACOBIAN OF PARALLEL ROBOTS
}

\author{
Jean-Pierre Merlet \\ INRIA \\ Sophia-Antipolis, France \\ Jean-Pierre.Merlet@sophia.inria.fr \\ Peter Donelan \\ Victoria University \\ Wellington, New-Zealand \\ Peter.Donelan@mcs.vuw.ac.nz
}

\begin{abstract}
Checking the regularity of the inverse jacobian matrix of a parallel robot is an essential element for the safe use of this type of mechanism. Ideally such check should be made for all poses of the useful workspace of the robot or for any pose along a given trajectory and should take into account the uncertainties in the robot modeling and control. We propose various methods that facilitate this check. We exhibit especially a sufficient condition for the regularity that is directly related to the extreme poses that can be reached by the robot.
\end{abstract}

Keywords: inverse jacobian, singularity, parallel robots

\section{Introduction}

Determining if a parallel robot may be in a singular configuration during its motion is a problem that is of high practical interest. Many papers have addressed first the determination of the inverse jacobian, denoted $\mathbf{J}^{-1}$, of such robots and then the analysis of the singularity condition that can be deduced from the singularity of this matrix. $\mathbf{J}^{-1}$ relates the joint velocities to the twist of the end-effector and is usually pose dependent. In a singularity the end-effector will exhibit non-zero velocities for some motion although the actuators are locked. The determinant of $\mathbf{J}^{-1}$ is usually complicated but for most parallel robots $\mathbf{J}^{-1}$ has as rows the Plücker vectors of well-defined lines. Consequently Grassmann geometry may be used to characterize the geometry of the singularity and to deduce simplified singularity conditions [Monsarrat 01; Merlet 89; Wolf 04]. It must be noted that even for robot with less than 6 d.o.f. it is necessary to consider the full jacobian matrix i.e. the matrix that involves the full twist of the end-effector. Indeed for a robot with $n$ d.o.f. 
the jacobian that relates the $n$ d.o.f. velocities to the $n$ actuated joint velocities may be not singular while $\mathbf{J}^{-1}$ is singular [Bonev 01].

A singularity detection algorithm should be able to determine the presence of a singularity within a motion variety with dimension 1 to $n$ for a $n$ d.o.f. robot. An important point is that the singularity detection should be certified i.e. the algorithm should provide a safe answer even if numerical round-off errors occur. This certification constraint usually rules out the use of an optimization procedure.

\section{A singularity detection scheme}

This singularity detection problem has been addressed in [Merlet 01] where an efficient algorithm was exhibited. This algorithm proceeds along the following steps: symbolic computation is used to determine an analytical form of the determinant of $\mathbf{J}^{-1}$ and its sign at a particular pose $\mathbf{X}_{\mathbf{1}}$. Then an interval analysis based method [Jaulin 01; Moore 79], that takes round-off errors into account, allows one to determine if the motion variety includes a set of poses in which the determinant has a sign opposite to the one found at $\mathbf{X}_{\mathbf{1}}$.

The main difficulty with this algorithm (apart of using efficiently interval analysis) is the calculation of the closed-form of the determinant as will be illustrated on a difficult example, the Gough platform.

\subsection{The inverse jacobian of a Gough platform}

We define a reference frame $(O, \mathbf{x}, \mathbf{y}, \mathbf{z})$. The attachment points of the leg $i$ on the base will be denoted by $A_{i}$. The attachment points on the platform will be denoted by $B_{i}$ and it is well known that the coordinates of $B_{i}$ in the reference frame can be obtained as function of the pose parameters. The inverse jacobian matrix is then constituted of the normalized Plücker vectors of the line associated to each leg:

$$
\mathbf{J}^{-1}=\left(\left(\frac{\mathbf{A}_{\mathbf{i}} \mathbf{B}_{\mathbf{i}}}{\left\|\mathbf{A}_{\mathbf{i}} \mathbf{B}_{\mathbf{i}}\right\|} \quad \frac{\mathbf{O A}_{\mathbf{i}} \times \mathbf{O B}_{\mathbf{i}}}{\left\|\mathbf{A}_{\mathbf{i}} \mathbf{B}_{\mathbf{i}}\right\|}\right)\right)
$$

Note that we may use the non normalized Plücker vector to define an-

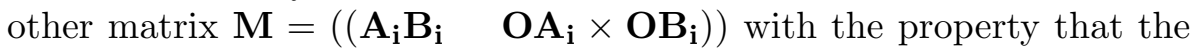
sign of $\mathbf{J}^{-1}$ is the same than those of $|\mathbf{M}|$. As $\mathbf{M}$ is simpler than $\mathbf{J}^{-1}$ it will be used for the singularity detection.

\subsection{Evaluation of the determinant}

Being given a motion variety the pose parameters are functions of the variety parameters and thus the components of the inverse jacobian may be obtained as functions of the variety parameters. As mentioned earlier 
a closed-form of the determinant is obtained by symbolic computation. It should be noted that this is not strictly necessary. Indeed being given ranges for the variety parameters interval arithmetic may used to determine ranges for each component of the inverse jacobian. We get then an interval matrix $\mathbf{J}_{I}^{-1}$ i.e. a matrix whose components are intervals. Classical method for the calculation of determinant may then be used to obtain an interval evaluation of the determinant but with a large overestimation of the minimum and maximum of the determinant. Indeed interval arithmetic is very sensitive to multiple occurrence of the same variable. Consider for example the matrix $\mathbf{A}$ whose determinant is $x y$ and its interval version $\mathbf{A}_{I}$ when $x$ and $y$ lie in the range $[1,2]$

$$
\mathbf{A}=\left(\begin{array}{cc}
x & x \\
y & 2 y
\end{array}\right) \quad \mathbf{A}_{I}=\left(\begin{array}{cc}
{[1,2]} & {[1,2]} \\
{[1,2]} & {[2,4]}
\end{array}\right)
$$

The interval evaluation of $\left|\mathbf{A}_{I}\right|$ may be calculated as $[-2,7]$. Hence the closed-form of the determinant allows one to show that $|\mathbf{A}|$ will always be positive for any value of $x, y$ in $[1,2]$, while the use of the interval matrix does not allow such conclusion. We have put an emphasis on interval matrices that will be justified by the influence of uncertainties.

\subsection{The influence of uncertainties}

Uncertainties are inherent part of a real system such as a robot. They occur at the modeling level: the geometry of the real robot differs from its theoretical model due to the manufacturing tolerances (for example for the Gough platform the locations of the $A_{i}, B_{i}$ are known only up to a known accuracy). Uncertainties are also due to control: there will be a deviation of the robot motion from the theoretical motion variety.

An ideal singularity detection scheme should be able to determine if the robot may be in a singular pose in spite of these uncertainties. Although we may add the uncertainties as additional unknowns in the components of $\mathbf{J}^{-1}$, a drawback is that the calculation of the closed-form of the determinant may become difficult. For example for the Gough platform Maple is no more able to calculate the determinant as soon as we add the uncertainties on the $A_{i}, B_{i}$. In that case we have to resort to a numerical interval evaluation of the determinant based on the interval version of $\mathbf{J}^{-1}$, but we have seen that this leads to a large overestimation of the determinant, that will result in a large computation time for the singularity detection scheme. It is thus necessary to develop methods that check the regularity of the set of matrices defined by an interval matrix, without calculating its determinant. These methods should take into account that $\mathbf{J}^{-1}$ is a parametric matrix, i.e. that its components are not independent. 


\section{Various methods for regularity check}

\subsection{A classical regularity check}

Checking the regularity of all matrices in a set defined by an interval matrix is a classical problem in interval analysis and is known to be NP-hard. Among possible approaches the one having shown the largest efficiency in our case has been a method proposed by Rohn [Kreinovich $00]$. We define the set $H$ as the set of all $\mathbf{n}$-dimensional vector $\mathbf{h}$ whose components are either 1 or -1 . For a given box we denote by $\left[a_{i j}, \overline{a_{i j}}\right]$ the interval evaluation of the component $J_{i j}^{-1}$ of $\mathbf{J}^{-\mathbf{1}}$ at the i-th row and j-th column. Given two vectors $\mathbf{u}, \mathbf{v}$ of $H$, we then define the set of matrices $\mathbf{A}^{\text {uv }}$ whose elements $A_{i j}^{\text {uv }}$ are

$$
A_{i j}^{\mathrm{uv}}=\overline{a_{i j}} \text { if } u_{i} \cdot v_{j}=-1, \underline{a_{i j}} \text { if } u_{i} \cdot v_{j}=1
$$

These matrices have thus fixed numerical components corresponding to lower or upper bound of the interval $J_{i j}^{-1}$. There are $2^{2 n-1}$ such matrices since $\mathbf{A}^{\mathbf{u v}}=\mathbf{A}^{-\mathbf{u},-\mathbf{v}}$. If the determinant of all these matrices have the same sign, then all the matrices $\mathbf{A}^{\prime}$ whose components have a value within the interval evaluation of $J_{i j}^{-1}$ are regular. Hence for the $6 \times 6$ $\mathbf{J}^{\mathbf{- 1}}$ of a Gough platform if the determinant of the 2048 matrices of $\mathbf{A}^{\mathbf{u v}}$ have the same sign, then all matrices in the set are regular.

But $\mathbf{A}^{\mathbf{u v}}$ includes matrices that are not inverse jacobian as the dependency of the components of the matrix are not taken into account. This may be seen, for example, for the interval matrix $\mathbf{A}_{I}$ (2) that includes the following matrices

$$
\mathbf{A}_{1}=\left(\begin{array}{ll}
1 & 1 \\
1 & 4
\end{array}\right) \quad \mathbf{A}_{2}=\left(\begin{array}{ll}
1 & 2 \\
2 & 2
\end{array}\right) \quad \mathbf{A}_{3}=\left(\begin{array}{ll}
1 & 2 \\
1 & 2
\end{array}\right)
$$

The matrices $\mathbf{A}_{\mathbf{1}}, \mathbf{A}_{\mathbf{2}}$ belong to the set $\mathbf{A}^{\mathbf{u v}}$ and have determinants with opposite signs. Consequently the test proposed by Rohn fails, which is quite normal as the matrix $\mathbf{A}_{\mathbf{3}}$, that belongs to $\mathbf{A}_{I}$ is singular. For the Gough platform the first column of $\mathbf{J}^{-\mathbf{1}}$ is written as $x+F_{i}, x$ being a coordinate of the center of the platform; if the range for $x$ is $[\underline{x}, \bar{x}]$ while the range for $F_{i}$ is $\left[a, b_{i}\right]$, then $\mathbf{A}^{\mathbf{u v}}$ includes matrices with elements $\underline{x}+a_{i}$ and $\bar{x}+b_{k}$ that does not belong to the set of inverse jacobian matrices.

\subsection{Pre-conditioning}

A classical approach in interval analysis for regularity check is to precondition the matrix by multiplying it by a real matrix $\mathbf{K}$, usually the inverse of the mid-matrix, i.e. the matrix whose components are the midpoint of each range of the components. The purpose of this strategy is 
to get $\mathbf{S}=\mathbf{K} \mathbf{J}^{-\mathbf{1}}$ close to the identity matrix so that its determinant $|\mathbf{S}|=|\mathbf{K}|\left|\mathbf{J}^{-\mathbf{1}}\right|$ may be interval evaluated with a lower overestimation. If we apply this strategy to the matrix (2) the inverse of the mid-matrix and the interval matrix $\mathbf{K} \mathbf{A}_{I}$ are:

$$
\mathbf{K}=\left(\begin{array}{cc}
4 / 3 & -2 / 3 \\
-2 / 3 & 2 / 3
\end{array}\right) \mathbf{S}=\mathbf{K} \mathbf{A}_{I}=\left(\begin{array}{cc}
{[0,2]} & {[-4 / 3,4 / 3]} \\
{[-2 / 3,2 / 3]} & {[0,2]}
\end{array}\right)
$$

The interval evaluation of $|\mathbf{S}|$ is $[-8 / 9,44 / 9] \approx[-0.8889,4.88889]$ while $|\mathbf{K}|$ is positive. In term of sign determination this interval evaluation is indeed sharper than the one obtained with a direct evaluation of $|\mathbf{A}|$, but is still not satisfactory. We propose another method which consists first to compute symbolically the matrix $\mathbf{S}$, using $k_{i j}$ as components of $\mathbf{K}$ and then plugging in the numerical values. The symbolic matrix $\mathbf{S}_{s}=\mathbf{A K}$ and its interval version $\mathbf{S}_{K}$ for the numerical $\mathbf{K}$ are

$$
\mathbf{S}_{s}=\left(\begin{array}{cc}
x\left(k_{11}+k_{21}\right) & x\left(k_{12}+k_{22}\right) \\
y\left(k_{11}+2 k_{21}\right) & y\left(k_{12}+2 k_{22}\right)
\end{array}\right) \mathbf{S}_{K}=\left(\begin{array}{cc}
2 x / 3 & 0 \\
0 & 2 y / 3
\end{array}\right)
$$

If we use now the range [1,2] for $x, y$ the interval evaluation of $|\mathbf{S}|$ is $[4 / 3,8 / 3]$ that shows that all matrices have a positive determinant. Note that we have used $\mathbf{A K}$ instead of $\mathbf{K A}$, which is justified as it allows to reduce the multiple occurrences of the variables. However as $\mathbf{J}^{-1}$ exhibits the same variables in a column it is better to pre-multiply it by the conditioning matrix.

\subsection{A regularity test for parametric matrices}

Assume that some components of some rows (denoted the linear rows) of a parametric matrix $\mathbf{A}=a_{i j}$ can be written as linear combination with real or interval coefficients of a set of unknowns $\left\{x_{1}, x_{2}, \ldots, x_{n}\right\}$.

We denote by $\mathbf{A}^{\prime}$ the set of real or interval matrices that can be derived from $\mathbf{A}$ by assigning independently to each linear rows either a lower or upper bound to each unknown $x_{i}$ that appears in the linear combination. For example for matrix $\mathbf{A}$ the set $\mathbf{A}^{\prime}$ is

$$
\mathbf{A}^{\prime}=\left\{\left(\begin{array}{ll}
1 & 1 \\
1 & 2
\end{array}\right),\left(\begin{array}{ll}
1 & 1 \\
2 & 4
\end{array}\right),\left(\begin{array}{ll}
2 & 2 \\
1 & 2
\end{array}\right),\left(\begin{array}{ll}
2 & 2 \\
2 & 4
\end{array}\right)\right\}
$$

The following theorem hold:

Theorem 1: If the determinant of all matrices in the set $\mathbf{A}^{\prime}$ have all the same sign, then all matrices in the set $\mathbf{A}$ are regular

Proof (derived from [Popova 04]): Assume that there is a singular matrix $\mathbf{A}_{\mathbf{0}}$ in the set $\mathbf{A}$. Without lack of generality we will assume that 
the first row of $\mathbf{A}_{\mathbf{0}}$ is linear. We consider the unknown $x_{1}$, whose value for $\mathbf{A}_{\mathbf{0}}$ is $x_{1}^{0}$ and lie in $\left[\underline{x_{1}}, \overline{x_{1}}\right]$. Each component of the first row of $\mathbf{A}$ may be written either as $\overline{\lambda_{1 j}^{1}} x_{1}+b_{1 j}$ or $a_{1 j}^{0}$ if the component is not linear. Using row expansion the determinant of the matrix may be written as

$$
|\mathbf{A}|=\sum_{k=j_{1}, \ldots, j_{m}}(-1)^{k+1}\left(\lambda_{1 k}^{1} x_{1}+b_{1 k}\right) M_{1 k}+\sum_{l \notin\left\{j_{1}, \ldots, j_{m}\right\}}(-1)^{l+1} a_{1 l} M_{1 l}
$$

where $\left\{j_{1}, \ldots, j_{m}\right\}$ are the column indices of the linear components of A and $M_{1 j}$ denotes the minor associated to the first line and column $j$.

For $x_{1}=x_{1}^{0}$ this expression will cancel. If we assume now that $x_{1}=$ $x_{1}^{0}+d x_{1}$ we get

$$
|\mathbf{A}|=d x_{1}\left(\sum_{k=j_{1}, \ldots, j_{m}}(-1)^{k+1} \lambda_{1 k}^{1}\right)=d x_{1} K_{1}
$$

$K_{1}$ being either a real number or an interval. We may always assign $d x_{1}$ to either $\bar{x}_{1}-x_{1}^{0}$ or $x_{1}-x_{1}^{0}$ so that $|\mathbf{A}|$ is positive or has a positive upper bound. Thus by assigning $x_{1}$ or $\overline{x_{1}}$ to $x_{1}$ we have constructed a matrix $\mathbf{A}_{1}^{+}$whose determinant will be positive or has a positive upper bound. The process may be repeated for constructing a matrix $\mathbf{A}_{1}^{-}$whose determinant will be negative or has a negative lower bound. Starting from these matrices we may now assign $x_{2}$ to $\underline{x_{2}}$ or $\overline{x_{2}}$ to get a matrix $\mathbf{A}_{12}^{+}$ whose determinant is $\left|\mathbf{A}_{1}^{+}\right|$plus a positive quantity (i.e. still positive) and a matrix $\mathbf{A}_{\mathbf{1 2}}^{-}$whose determinant will be lower than the determinant of $\left|\mathbf{A}_{1}^{-}\right|$(i.e. still negative). The process is repeated for each unknowns in the row. As soon as all unknowns in the row have a fixed value the process is repeated for the next linear row. When all linear rows have been processed the matrices $\mathbf{A}^{+}, \mathbf{A}^{-}$belong to $\mathbf{A}^{\prime}$. Note however that the assignment of the unknowns in a row to ensure that $\left|\mathbf{A}^{+}\right|$is positive may differ between two linear rows. Hence if there is a singular matrix in $\mathbf{A}$, then we are able to determine matrices whose determinant have opposite signs (or whose lower bound is negative and upper bound is positive), which concludes the proof.

For example as all matrices in $\mathbf{A}^{\prime}$ defined by (6) have the same determinant sign, then the set $\mathbf{A}$ contains only regular matrices. Another theorem may be derived for the full inverse jacobian matrices that have Plücker vectors as rows. Let us define $A_{i}\left(a_{i}^{1}, a_{i}^{2}, a_{i}^{3}\right)$ and $B_{i}\left(b_{i}^{1}, b_{i}^{2}, b_{i}^{3}\right)$ as two points that belong to the line associated to the Plücker vector $i$. A row of $\mathbf{J}^{-1}$ may be written as

$$
\left(\left(b_{1}-a_{1}, b_{2}-a_{2}, b_{3}-a 3, a_{2} b_{1}-a_{1} B_{2}, a_{3} b_{1}-a_{1} b_{3}, a_{1} b_{2}-a_{2} b_{1}\right)\right)
$$

so that each row is linear in the $b_{i}$. Assume now that the locations of the $A_{i}$ are fixed, while the locations of the $B_{i}$ are functions of the endeffector motion. Using interval analysis (or an optimization method) 
being given ranges for the motion parameter we may find a bounding box $\mathcal{B}_{i}$ for the location of each $B_{i}$. Let $\mathbf{J}_{\star}^{-\mathbf{1}}$ be the set of inverse jacobian that may be obtained for the motion parameters ranges. Theorem 1 allows one to state the following theorem:

Theorem 2: Let $\mathbf{A}^{\star}$ be the set of matrices obtained by choosing as location of $B_{i}$ all possible combinations of the corners of $\mathcal{B}_{i}$ (there will be $8^{6}$ such matrices). If the determinants of all matrices in $\mathbf{A}^{\star}$ have the same sign, then all matrices in $\mathbf{J}_{\star}^{-\mathbf{1}}$ are regular.

The number of matrices in $\mathbf{A}^{\star}$ may even be reduced in some cases, using the property that we may choose as $B_{i}$ any point on the line. Assume that the bounding box $\mathcal{B}_{i}$ is defined by the set of ranges $\left[\underline{b_{i j}}, \overline{b_{i j}}\right]$, $j \in[1,3]$ for $b_{j}$. The following cases may occur:

- $a_{k} \in\left[b_{i k}, \overline{b_{i k}}\right]$ for two indices in $[1,2,3]$, while $a_{k}<b_{i k}$ or $a_{k}>\overline{b_{i k}}$ for one index. The line always enters the bounding box $\mathcal{B}_{i}$ by the face defined by $b_{k}=. b_{i k}$ or $b_{k}=\overline{b_{i k}}$. We may thus choose as $B_{i}$ the intersection point of the line with this face i.e. fix the value of $b_{k}$. Hence only 4 corners will have to be checked

- $a_{k} \in\left[\underline{b_{i k}}, \overline{b_{i k}}\right]$ for only one index. The line may enter the bounding box by 2 faces and we have to check 6 corners

- $a_{k} \notin\left[\underline{b_{i k}}, \overline{b_{i k}}\right]$ for all index. The line may enter the bounding box by 3 faces and we have 7 corners to check

- $a_{k} \in\left[b_{i k}, \overline{b_{i k}}\right]$ for all index. In that case the corresponding row of the jacobian may include a line of 0 and the ranges for the motion parameters must be bisected

In practice we will have between $4^{6}$ and $7^{6}$ matrices in $\mathbf{A}^{\star}$. Uncertainties in the locations of the $A_{i}$ may also be dealt with by considering that the matrices in $\mathbf{A}^{\star}$ are interval matrices.

Theorem 2 shows that checking the extreme poses of the $B_{i}$ may be sufficient to check the regularity of $\mathbf{J}^{-1}$ over the whole workspace.

\section{Examples}

The proposed regularity check has been implemented in the singularity detection scheme and has been extensively tested. It appears that among the three regularity checks the most efficient combination is to use first the pre-conditioning and then to apply Rohn test on the resulting matrix. A $6 \mathrm{D}$ workspace $\mathcal{W}$ is defined with the ranges $x, y$ in $[-15,15]$, $z$ in $[45,50]$ and the three Euler angles having the ranges $[-15,15]$ degree. The computation time on a Dell D400 laptop (1.7 Ghz) is established as follows: 
- 6D workspace without uncertainty: for $\mathcal{W}$ no singularity detected in $3.12 \mathrm{~s}$. If the orientation ranges of $\mathcal{W}$ is extended to $[-40,40]$ degree a singularity is detected in $9.46 \mathrm{~s}$.

- $6 \mathrm{D}$ workspace with uncertainties: for a \pm 0.05 uncertainty on each coordinates of the $A_{i}, B_{i}$ points no singularity is detected in $\mathcal{W}$ in $43 \mathrm{mn}$ on a cluster of 15 PC's without the regularity checks and only in $263 \mathrm{~s}$ on a laptop if they are incorporated in the detection scheme. For an uncertainty of \pm 0.1 the computation time establishes respectively at 10h $22 \mathrm{mn}$ and $1176 \mathrm{~s}$.

\section{Conclusion}

We have proposed regularity checks for the inverse jacobian of parallel robots that may be used to determine if such matrix may be singular over a motion variety. They allow to deal with uncertainties in the robot modeling and control and have been proved to be very efficient. One of this regularity check, that is sufficient but not necessary, is related to the extremal poses that can be reached by the end-effector: if the determinant of a finite number of real matrices that are related to these extremal poses have all the same sign, then the inverse jacobian matrix is regular.

\section{References}

Bonev I.A. and Zlatanov D. The mystery of the singular SNU translational parallel robot. www.parallemic.org/Reviews/Review004.html, June, 12, 2001.

Jaulin L., Kieffer M., Didrit O., and Walter E. Applied Interval Analysis. SpringerVerlag, 2001.

Kreinovich V. Optimal finite characterization of linear problems with inexact data. Technical Report CS-00-37, University of Texas at El Paso, 2000.

Merlet J-P. Singular configurations of parallel manipulators and Grassmann geometry. Int. J. of Robotics Research, 8(5):45-56, October 1989.

Merlet J-P. and Daney D. A formal-numerical approach to determine the presence of singularity within the workspace of a parallel robot. In F.C. Park C.C. Iurascu, editor, Computational Kinematics, pages 167-176. EJCK, Seoul, May, 20-22, 2001.

Monsarrat B. and Gosselin C.M. Singularity analysis of a three-leg six-degree-offreedom parallel platform mechanism based on Grassman line geometry. Int. J. of Robotics Research, 20(4):312-328, April 2001.

Moore R.E. Methods and Applications of Interval Analysis. SIAM Studies in Applied Mathematics, 1979.

Popova E. D. Strong Regularity of Parametric Interval Matrices 33th Spring Conference of the Union of Bulgarian Mathematicians, Borovets, April 1, 2004

Wolf A. and others. Application of line geometry and linear complex approximation to singularity analysis of the 3-dof CaPaMan manipulator. Mechanism and Machine Theory, 39(1):75-95, January 2004. 